\title{
Introduction to Practice-based IS Research Minitrack
}

\author{
Dorothy Leidner \\ Baylor University \\ dorothy_leidner@baylor.edu \\ Ester Gonzalez \\ California State University, Fullerton \\ esgonzalez@fullerton.edu
}

\author{
Bill Kettinger \\ University of Memphis \\ wjkttngr@memphis.edu \\ Michael Milovich, Jr. \\ Rowan University \\ milovich@rowan
}

This minitrack seeks to encourage practice-based research on new and emerging IS issues in organizations. Practice-based research aspires to bridge the gap between academic theory and practice; it aspires both to introduce researchers to state of the art practices and issues from industry as well as introduce managers to research that makes sense of and brings coherence to the issues they face. The methods used in practice-based research are often exploratory, fieldbased studies involving interviews, observations, and/or descriptive surveys. The intense pressure to achieve methodological distinction and theoretical contribution often results in very current practice-based topics being eschewed by researchers, because the topics themselves are not mature enough in practice to achieve desirable samples or sample sizes, nor are they conducive to theorizing since so little is known. These are precisely the reasons that exploratory, practicebased research can play a tremendous role in helping establish and lay the foundations of a research domain while providing insights into an emerging topic.

The objective of this minitrack is to encourage practice-based research in information systems and to disseminate the results of that research in a manner that makes its relevance and utility readily apparent. This minitrack invites authors to submit in-depth research that provides rich stories, unique insights, and useful conceptual frameworks for information systems practice. Papers might be based upon single cases, multiple case, field interviews, or, less commonly, literature itself. Experimental research and survey research are less likely to achieve the goal of providing rich insight for practice. While it is assumed that researchers are guided by theory, it is not expected that the submissions to this minitrack make distinct or novel theoretical contributions. The contributions should focus on distinct and unique lessons for practice. The minitrack chairs coordinates with MIS Quarterly Executive in selecting papers for fasttracking to an issue of MISQE.

Overall, this minitrack aims to:

- Showcase high quality practice-oriented IS research

- Promote practice-oriented IS research as a key source of insight and guidance for digital leaders

- $\quad$ Provide researchers a platform to present and discuss their practice-oriented IS research findings and expose the community to current challenges in creating value with IT

- Help identify the most challenging managerial issues for digital/IT leaders and frame them as new questions that could guide future practiceoriented IS research. 